\title{
VIII. Feuilleton.
}

\section{Ein Arzneibuch von 1678.}

Von Dr. R. Landau, prakt. Arzt in Frankenberg i. S.

Wer für die Wissenschaft, die er in seinem Berufe vertritt, wahres Interesse hegt, der wird auch gern von der Vergangeubeit derselben sich unterrichteu lassen, und darum hoffe ich einigen Beifall zu finden, wenn ich meinen Collegen eine kurze Mittheilung über ein altes medicinisches Buch, das mir ein Zufall in die Hände spielte, gebe.

Das Buch ist betitelt „Erneuerte Hauss- und Feld-Apothek oder Stadt- und Laud-Artzney-Buch, darinnen zu finden wie sich der Mensch beydes vor allerhand Zufälle, als auch in denenselben verbalten solle; da dann die Recepten dergestalt eingerichtet, dass sowobl der Stadt- als Landmann sein Genügen daran haben und selbige nach seinem Belieben erwehlen kanu. Hiebevor an den Tag gegeben von Carl de Gogler; nunmebr aber in vielen verbessert und durch eine richtige Ordnung in unterschiedene Theile zusammengetragen - Frankfurt am Main, In Verlegung Martin Halle vor den Buchh., in Königsberg getruckt bey Johann Andreae, im Jahre 1678." Auf dem Titelbild empfängt ein an einem Krankenbett stehender Arzt aus der Hand einer ältlichen Frau einen Bund Kräuter.

Was die richtige Ordnung " anlangt, so ist dieselbe nach den Krankheiten festgesetzt, und diese wieder scheiden sich nach den Körpertheilen in absteigender Reihe. Also handelt der Verfasser zuerst , ,on den Kranckheiten des Hauptes und Halses“. Da giebt es z. B. "ein Säcklein für Wehetag des Haupts von Frau Elisabeth Gräfin von Schwartzenburg", eine grosse Anzabl Recepte "vor die Flüsse des Kopffs" und "vor die Flüsse dess halben Haupts", ferner "vor die hinfallende Kranckheit oder schwere Notb" (darunter eines "der alten Churfürstin von Weimar" und eines , von der gefangenen Hertzogin von Oesterreich vor den Schlag“; , vor die Gicht"; "für den Schwindel"; , vor die Melancholey "; "vor die Unsinnigkeit"; „zu der Gedächtniss"; „vor die Schlafsucht"; „wenn einer nicht schlafen kann"; dazu kommen noch Mittel gegen ObrenNasen- und Augenkrankbeiten, gegen Zahnweb, ,vor die Bräune", "vor die Drüsen in dem Hals", "für die Geschwühr an der Keblen" "vor die Heiserkeit des Halses und der Kehlen". Der zweite Theil enthält die Behandlung der Krankheiten der Brust, und wir finden da im bunten Durcheinander ein Kapitel "von den Kranckheiten des Hertzens und Ohnmachten", eines "vor das Seitenstechen", eines "vor Geschwühr in der Seiten", ein viertes, vor Brustgeschwübr", ein fünftes "vor den Husten", weiter Kapitel, die Mittel "für Engbrüstigkeit", ", or die Lungensucht", , vor das Aufsteigen "der Iungen", "vor Lungen- und Leberfäulung " enthaltend. Im dritten Theil, welcher "von den Kranckheiten des Bauchs" handelt, giebt es Heilmittel "vor einem blöden Magen“, "vor Geschwühr des Magens", "vor das Erbrechen", , vor den unersättlichen Hunger oder Vielfrässigkeit", , vor den Durst", „vor den Sodt", „vor die Winde im Leib", vor Verstopffung des Leibs" und vor den Durchbruch des Leibs", "vor die Wassersucht"; aber auch Leber, Niere, Milz sind von dem Verfasser bedacht worden, und zum Schluss kommt ein Mittel "contra impotentiam" zur Empfehlung. Den vierten Theil bilden die "Kranckheiten der Glieder" (dabei spielt das Podagra die grösste Rolle), den fünften „die Kranckheiten der Weiber und Kinder“, wobei auch Ratschläge "vor die scbwere Geburt der Weiber" ertheilt werden. Mehr anhangsweise werden in den folgenden Theilen "die Kranckheiten der Fiebern und Vergifftungen" besprochen; dann bandelt der Verfasser "von allerhand Wunden", ferner "von dem Angesicht und Haar", endlich "von Purgantzen und Pflastern".

Ich habe diese Inhaltsübersicht absichtlich etwas ausführlich mitgetheilt, weil sie, wie mir scheint, einen Einblick in den Stand der damaligen Medicin gestattet. Wie nach rein äusserlicher, jeder wissenschaftlichen Vertiefung entbehrender Methode der Kopf eine Abtheilung giebt, die Brust eine zweite u. s. f., so finden sich in 
den folgenden grossen Theilen und in den einzelnen Kapiteln aller dieser Theile als Krankheiten Symptome der verschiedensten Krankbeiten genannt (Husten, Wassersucht, Fieber etc.), und so wird Chirurgie, interne Medicin und Geburtshülfe wirr und wild durch einander gew orfen.

Sehr merkwürdig ist es, um dies noch zu erwähnen, gewiss für unsere moderne medicinische Bildung, zu bören, dass sich die antiluetische Cur (, , vor die Frantzosen ${ }^{\circ}$ ) in dem siebenten Theil , von allerhand Wunden" beschrieben findet. Wird so die vollständig un wissenschaftliche, jeder Kritik mangelnde Art der damaligen Medicin in das hellste Licht gesetzt, so zeigt sich im übrigen ein Wust von blindern Aberglaubeu, der fast kindlich zu nennen ist.

Das ergiebt sich vor allem aus der Art der angepriesenen Mittel und vielleicht noch mehr aus der Anwendungsweise dieser Mittel.

Wie bekannt und wie leicht erklärlich, wurden die Heilmittel vor zweihundert Jahren hauptsächlich aus der Pflauzenwelt entnommen. Es ist ein interessanter Beitrag zur Gescliichte der Medicin und insbesondere zur Geschichte der Pharmakologie, pflanzliche Producte in diesem alten Arzneibuche 21 finden, welche auch unser, deutsches Arzneibuch" vom Jalıre 1811 entlıält. Um einiges zu nennen, greife ich aus den angegebenen Recepten beliebig heraus: Süssholz, Eibischwurzel, Sennesblätter, Cubeben, Zimmtrinde, Rhabarber, Aloë, Baldrianwurzel, Tamarinden, Wacliholderbeeren, Herba Absynthii, Bilsensamen und Bilsenkraut, Mobnblüthen, Farrenkrant (Asepidion Filix), Lignum Guajaci, Asa fötida etc. Dazı kommen von pflanzlichen Producten Campher, Terpenthin, Meerzwiebelessig, Rosenwasser, Fenchelwasser, Süss- und Bittermandelöl, Gummi arabicum u: s: w. Zu "Schlaffpillen ${ }^{*}$ diente auch damals "Laudanum opiatum “. Die übrigen Kräuter, die sich in unserer Pharmakopoe. nicht erhalten baben, zu nennen, böte zu wenig Interesse und würde zu "weit fïhren; es findet sich bier etwa alles, was noch beute in der botanischen Sprache den Beinamen ,officinalis“ führt.

Aus dem Thierreich wurden, wie noch heute, schon damals die Canthariden verwendet; viele Recepte enthalten Castoreum. Aber ausser diesen beiden. Producten lieferte die Fauna die glaublichsten und unglaublichsten Mittel, und in ihneu gerade lässt sich oft der mystische Zug erkennen, der die damalige Pharmakopoe durchwehte. Ausser Schweineschmalz wurde äusserlich, insbesondere zu Salben, Bärenschmalz, Hundsschmalz, „Fett von einer Wachtel “ und ähnliches verbraucht. Nicht nur äusserlich, sondern auch iunerlich nahm man "Pferdsdreck" - z. B. heisst es: "wer mit Grimınen (des Bauchs) beladen, der nehme, wann es ihnen ankommt, frischen Pferdsdreck, drück den Saft darauss in einem reinen Tiichlein, vermenge denselben mit ein wenig warm Bier, und trinke es ein, so wird er alsbald Besserung befinden. Probatum est." Rindermist mit Essig zu einem Pflaster verarbeitet, diente zur Blutstillung, ,wann einem die Adern zerhauen sind “. "Ein herrlich Wasser für den Stein" wird durch Destillation von "Taubenmist, der schön ist, er sey dörr oder feucht" und Petersilie in Wein dargestellt. Nasenbluten stillt "Saumist" ${ }^{\alpha}$, röste ihn in einer Pfannen und halte es dem Blutenden vor die Nase titlichen Sachen bezeichnet die Vorschrift "so eine Frau hart zur Geburt arbeitet, gebe ihr ihres Mannes Harn zu trinken. Probatum est. * Menschlicher Urin wird übrigens noch einmal empfohlen, und zwar soll man Rauten, Fencliel, Endividien u. s. w. zerstossen, zwölf Stunden in Wein legen und darauf, 24 Stunden in den Harn eines Kinds von zehn Jahren", um ,ein Mahl zu vertreiben und das Gesicht zu erklären". Auf Widerstand würden wir wohl auch' bei unseren Patienten stossen, wollten wir ihnen Hirnschädel von einem gerichteten Mann" verordnen. In dem Mittel "vor die hinfallende Krankheit von der gefangenen Hertzogin von "Oesterreich" heisst es z. B. "Rp. Des gefeileten Hirnschedels von einem geköpfften Mann $\bar{z}_{i}$ (wann der Mensch weiblichen Geschlechts ist, muss von dem Schedel eines geköpfften Weibs genommen werden), geschälte Peonienkörner num. CXX, mische es unter einauder, uud gebe abends und morgends auff einen Monat $3 \mathrm{i}$ mit Lindenblutwasser dem Krancken ein". Weitere Heilmittel bietet die Thierwelt in der "Kranichsgall“, der „Schweinemilch“, der „Pferdsmilclł“ (,einem "Weib unwissend eingegeben kurtz vor den ehelichen Wercken macht sie fruchtbar"), dem ,weissen Haasenhaar, so er unten auf der Bauchhaut bat", dem "Igelfleisch oder dessen Leber gedörrt und mit Honigsyrup eingenommen", der Fuchslunge nnd der Wolfsleber, "Wie man die Wolffsleber brauchen solle", lehrt ein besonderes Kapitel; "wider die Faulung oder Geschwühre der Lunge und Leber, davon die Lungensucht herkommt, ist die Wolffsleber sehr gut und nützlich," heisst es daselbst. Doch muss man bei Männern die Leber eines Wolfs, bei Weibern die einer Wölfin nehmen; jedenfalls muss man die frische Leber in einen Backofen backen, „dass sie fein dörr werde, doch dass sie nicht zu Kohlen verbrenne." Es sind weiter als Heilmittel genannt „Pulver von einer Kröten", „Cerebrum passerum", „Taubenhirn", mehrfach Re- genwürmer (,mit Wein sauber gewaschen und aufgedürret"), endlich „zerriebene Schaben, so Du im Keller findest"‘.

(Schluss folgt.) 